\title{
Intravenous Inoculation of Replication-Deficient Recombinant Vaccinia Virus DIs Expressing Simian Immunodeficiency Virus Gag Controls Highly Pathogenic Simian-Human Immunodeficiency Virus in Monkeys
}

\author{
Yasuyuki Izumi, ${ }^{1,2}$ Yasushi Ami, ${ }^{3}$ Kazuhiro Matsuo, ${ }^{1,2}$ Kenji Someya, ${ }^{1}$ Tetsutaro Sata, ${ }^{4}$ \\ Naoki Yamamoto, ${ }^{1}$ and Mitsuo Honda ${ }^{1,2 *}$ \\ AIDS Research Center, ${ }^{1}$ Division of Experimental Animal Research, ${ }^{3}$ Department of Pathology, ${ }^{4}$ National Institute \\ of Infectious Diseases, Shinjuku-ku, Tokyo 162-8640, and Japan Science and Technology Corporation, \\ Kawaguchi, Saitama 332-0012, ${ }^{2}$ Japan
}

Received 15 July 2003/Accepted 27 August 2003

\begin{abstract}
To be effective, a vaccine against human immunodeficiency virus type 1 (HIV-1) must induce virus-specific T-cell responses and it must be safe for use in humans. To address these issues, we developed a recombinant vaccinia virus DIs vaccine (rDIsSIVGag), which is nonreplicative in mammalian cells and expresses the full-length gag gene of simian immunodeficiency virus (SIV). Intravenous inoculation of $10^{6}$ PFU of rDIsSIVGag in cynomologus macaques induced significant levels of gamma interferon (IFN- $\gamma$ ) spot-forming cells (SFC) specific for SIV Gag. Antigen-specific lymphocyte proliferative responses were also induced and were temporally associated with the peak of IFN- $\gamma$ SFC activity in each macaque. In contrast, macaques immunized with a vector control (rDIsLacZ) showed no significant induction of antigen-specific immune responses. After challenge with a highly pathogenic simian-human immunodeficiency virus (SHIV), $\mathrm{CD}^{+} \mathrm{T}$ lymphocytes were maintained in the peripheral blood and lymphoid tissues of the immunized macaques. The viral set point in plasma was also reduced in these animals, which may be related to the enhancement of virus-specific intracellular IFN- $\gamma^{+} \mathrm{CD8}^{+}$cell numbers and increased antibody titers after SHIV challenge. These results demonstrate that recombinant DIs has potential for use as an HIV/AIDS vaccine.
\end{abstract}

Numerous studies have demonstrated that antiviral cellular immunity is critical for controlling replication of human immunodeficiency virus type 1 (HIV-1) in infected individuals (10, 22, 29 ) and for protecting monkeys from pathogenic challenge with simian immunodeficiency virus (SIV) $(2,5,38,43)$. The containment of primary infection is suggested to correlate with the induction of multivalent and high-affinity cytotoxic $\mathrm{T}$ lymphocytes (CTL) $(1,9,12,28)$ and enhanced chemokine production $(18,19)$. In addition, strong virus-specific helper T-cell responses are also believed to be critical for the induction and maintenance of effective protective immunity $(32,33,44,45)$.

To induce protective immunity, recombinant vaccinia virus strains (27), including modified vaccinia virus Ankara (MVA) (40) and a substrain of Copenhagen (NYVAC) (42), are currently being evaluated as recombinant vectors for HIV vaccines. Since these strains retain the ability to replicate under certain conditions and therefore may be potentially virulent, we explored the use of an alternate vaccinia virus strain, DIs, for use as a vaccine vector. This strain was developed more than 40 years ago $(17,41)$ and has been shown to be replication deficient in mammalian cells $(15,24)$.

At present, many candidate vaccines against HIV-1 utilize multicomponent viral proteins for the induction of strong HIV-specific immune responses. SIV vaccines expressing Gag,

\footnotetext{
* Corresponding author. Mailing address: AIDS Research Center, National Institute of Infectious Diseases, Shinjuku-ku, Tokyo 162 8640, Japan. Phone: 81-3-5285-1111, ext. 2737. Fax: 81-3-5285-1183. E-mail: mhonda@nih.go.jp.
}

Pol, Env, and regulatory proteins have been shown to induce efficient cellular immune responses and protect against pathogenic virus challenge in nonhuman primate models. These vaccine modalities consist of prime/boost regimens, including DNA/recombinant MVA with or without interleukin-2 $(2,5)$ and DNA/recombinant adenovirus (38). The potential of SIV candidate vaccines expressing single viral proteins has recently been reported with Manu-A*01 macaques receiving four inoculations with SIV Gag DNA (8) and with adenovirus type 5 vectors expressing the SIV Gag protein (38). These vaccines elicited immune responses able to control SIV or simian-human immunodeficiency virus (SHIV) infection in macaques.

In the present study, we constructed a recombinant vaccinia virus DIs expressing SIV Gag protein (rDIsSIVGag) and found that both DIs and recombinant DIs (rDIs) were replication deficient in mammalian cells. By comparison, MVA had significant levels of replication in these cells. Moreover, we found that the expression of Gag alone by rDIsSIVGag was sufficient to induce significant protection from pathogenic virus challenge in a SHIV/macaque model. Virus-specific immunity was elicited by two intravenous inoculations of the vaccine. Although rDIsSIVGag is replication defective in mammalian cells, it expresses SIV p27 antigen, suggesting a very safe and effective vector for HIV vaccine development.

\section{MATERIALS AND METHODS}

Macaques and SHIV challenge stocks. Cynomologus macaques (Macaca fascicularis) were maintained according to the institutional animal care and use guidelines of the National Institute of Infectious Diseases (NIID), Tokyo, Japan, 
and were free of known simian retroviruses, herpesviruses, bacteria, and parasites. The study was conducted in a biosafety level 3 facility at the Murayama Branch, NIID, under the approval of an institutional committee for biosafety and in accordance with the requirements of the World Health Organization. SHIV$\mathrm{C} 2 / 1$ was used as a challenge virus $(34,37,47)$ by intravenous inoculation of 20 $50 \%$ tissue culture infective dose(s) $\left(\right.$ TCID $\left._{50}\right)$; SHIV-C2/1 is an SHIV-89.6 variant isolated by passaging the peak of initial plasma viremia from an infected cynomologus macaque (37). The original SHIV strain was kindly provided by Y. $\mathrm{Lu}$ at the Harvard AIDS Institute (Boston, Mass.) (20, 31).

Cells. Cells were maintained in humidified air with $5 \% \mathrm{CO}_{2}$ at $37^{\circ} \mathrm{C}$. Human HeLa (ATCC CCL-2), HepG2, 293T, rabbit kidney RK13 (ATCC CCL-37), African green monkey CV-1 (ATCC CCL-70), Chinese hamster ovary $(\mathrm{CHO})$, and baby hamster kidney BHK-21 (ATCC CCL-10) cells were grown in Dulbecco modified Eagle medium supplemented with $10 \%$ fetal calf serum. Chicken embryo fibroblast (CEF) cells were grown in minimal essential medium supplemented with $5 \%$ fetal calf serum.

Production and preparation of rDIs expressing full-length SIV Gag. Detailed methods for plasmid construction were described previously (15). Briefly, CEF cells were grown in $8-\mathrm{cm}$ dishes and infected with DIs at a multiplicity of infection (MOI) of 1.0. Cells were transfected with $20 \mu \mathrm{g}$ of pUC/DIsLacZ by using Lipofectamine (Gibco-BRL/Life Technologies, Gaithersburg, Md.). rDIs expressing $\beta$-galactosidase (rDIsLacZ) was selected by four consecutive rounds of plaque purification in CEF cells stained with X-Gal (5-bromo-4-chloro-3indolyl- $\beta$-D-galactopyranoside; Takara). rDI virus expressing SIVmac239 Gag was obtained by using rDIsLacZ as the parental virus with the transfer vector pUC/DIsGag and then cloned as described above. Virus was grown in the chorioallantoic membrane of 10-day-old eggs or by culture with CEF cells. Virus preparations were purified by sucrose density gradient ultracentrifugation and adjusted to $10^{7} \mathrm{PFU} / \mathrm{ml}$. Methods for virus detection, culturing the recombinant clones, and Western blotting of cell lysate were performed as previously described $(15,23,24,26,41)$. The anti-SIV Gag monoclonal antibody (MAb) IB6 was kindly supplied by T. Sata, Department of Pathology, NIID, and by K. Ikuta, Institute for Microbial Diseases, Osaka University, Suita, Osaka, Japan (21). Primers used for PCR amplification were synthesized according to published sequences (15) and were designed to avoid destruction of any open reading frames. To insert the amplified fragments into pUC/DIsNot, an extra NotI site was added on the 5' termini of the primers. Amplified fragments were cut with Not I and inserted into the same site of pUC/DIsNot. These transfer vectors were used for the construction of rDIs by using rDIsLacZ as the parental virus. The recombinant viruses were propagated in CEF cells. Vaccinia virus strain WR, recombinant WRSIVGag, and MVA were kindly supplied by the Centralized Facility for AIDS Reagents, National Institute for Biological Standards and Controls, Potters Bar, Hertfordshire, United Kingdom. For analysis of virus replication, confluent monolayers of mammalian cells in 6-cm dishes were infected with the recombinant viruses at an MOI of 0.05 . After $1 \mathrm{~h}$ at $37^{\circ} \mathrm{C}$, the cells were washed with phosphate-buffered saline (PBS) and incubated in fresh medium at $37^{\circ} \mathrm{C}$. The cells were harvested at 0 and $48 \mathrm{~h}$ after absorption, freezethawed, and sonicated. Virus replication was determined by dividing the virus yield at $48 \mathrm{~h}$ by that at $0 \mathrm{~h}(15)$.

Virus-specific IFN- $\boldsymbol{\gamma}$ ELISPOT assay. Enzyme-linked immunospot (ELISPOT) assays were performed by the method and with the instruction of Mothe and Watkins, Wisconsin University Primate Center (28). In brief, 96-well flat-bottom plates (U-CyTech-BV, Utrecht, The Netherlands) were coated with anti-gamma interferon (IFN- $\gamma$ ) MAb MD-1 (U-CyTech-BV) overnight at $4^{\circ} \mathrm{C}$. The plates were then washed with PBS containing $0.05 \%$ Tween 20 (PBST) and blocked with PBS containing $2 \%$ bovine serum albumin (PBSA) for $1 \mathrm{~h}$ at $37^{\circ} \mathrm{C}$. PBSA was discarded from the plates. Freshly isolated PBMC were added with either concanavalin A (ConA) or a $0.2 \mu \mathrm{M}$ concentration of pooled Gag peptides (AIDS Research and Reference Reagent Program) and were then incubated for $16 \mathrm{~h}$ at $37^{\circ} \mathrm{C}$ in $5 \% \mathrm{CO}_{2}$ in anti-IFN- $\gamma$-coated plates, followed by a lysing step with ice-cold deionized water. After the plate was washed, rabbit anti-IFN- $\gamma$ polyclonal biotinylated detector antibody ( $1 \mu \mathrm{g} /$ well; U-CyTech-BV) was added, and the plates were further incubated for $1 \mathrm{~h}$ at $37^{\circ} \mathrm{C}$. The plates were then washed with PBST, after which $50 \mu$ l of gold-labeled anti-biotin immunoglobulin $\mathrm{G}$ (GABA) solution (U-CyTech-BV) was added, followed by incubation for $1 \mathrm{~h}$ at $37^{\circ} \mathrm{C}$. After a wash with PBST, activator mix (30 $\mu \mathrm{l} /$ well; U-CyTech-BV) was added, and the plates were allowed to develop for $15 \mathrm{~min}$.

Wells were imaged and spot-forming cells (SFC) were counted by using the KS ELISPOT compact system (Carl Zeiss, Jena, Germany). An SFC was defined as a large black spot with a fuzzy border (28). To determine significance levels, a baseline for each peptide was established by using the averages and standard deviations (SD) of the number of SFC for each peptide. A threshold significance value corresponding to this average plus two SD was then determined. A re- sponse was considered positive if the number of SFC exceeded the threshold significance level of the sample with no peptide.

Detection of intracellular IFN- $\boldsymbol{\gamma}$ by flow cytometry. Freshly isolated PBMC $(5 \times$ $10^{5}$ to $1 \times 10^{6}$ cells) were suspended in R-10 medium and incubated with antigen for $16 \mathrm{~h}$ at $37^{\circ} \mathrm{C}$ with $5 \% \mathrm{CO}_{2}$. For the final 6 to $8 \mathrm{~h}$, brefeldin A (Sigma Chemical Co., St. Louis, Mo.) was added at $10 \mu \mathrm{g} / \mathrm{ml}$. Antibody to CD28 (1 $\mu \mathrm{g} / \mathrm{ml}$; BD Pharmingen, San Diego, Calif.) was also added during the incubation as a costimulator molecule. After stimulation, the cells were washed and stained with fluoroscein isothiocyanate-conjugated anti-CD3 (FN18; Biosource, Camarillo, Calif.) and peridinin chlorophyll protein-conjugated anti-CD8 antibodies (Leu2a; Becton Dickinson). The cells were then sequentially incubated for fluorescence-activated cell sorting (FACS) lysing solution (Becton Dickinson Biosciences, San Jose, Calif.) for $10 \mathrm{~min}$ and FACS permeabilizing solution (Becton Dickinson) for another $10 \mathrm{~min}$. The cells were washed, stained with phycoerythrin-conjugated anti-human IFN- $\gamma$ antibody (4S.B3; BD Pharmingen), and fixed with $2 \%$ paraformaldehyde. Samples were analyzed with a FACSCalibur apparatus (Becton Dickinson) using CellQuest software (Becton Dickinson).

Lymphocyte proliferative responses. SIV-specific proliferative responses were measured in freshly isolated PBMC. In brief, $2 \times 10^{5}$ PBMC were resuspended in $200 \mu \mathrm{l}$ of growth medium and plated in flat-bottom 96-well plates with either ConA or purified SIVmac251 p27 protein (Advanced BioScience Laboratories, Rockville, Md.) (14). After 3 days of culture, $\left[{ }^{3} \mathrm{H}\right]$ thymidine was added to the wells, and the cells were harvested $16 \mathrm{~h}$ later to determine uptake.

Antibodies to SIV p27. Antibodies to SIV Gag p27 were detected as described previously (37). Briefly, recombinant SIV p27 protein (ImmunoDiagnostics, Inc., Woburn, Mass.) was coated on a microplate at a concentration of $2 \mu \mathrm{g} / \mathrm{ml}$. The mean antibody titer was expressed as the reciprocal of the serum dilution that exceeded the cutoff value by two SD.

Absolute $\mathrm{CD4}^{+}$- and $\mathrm{CD8}^{+}$-T-lymphocyte counts. An absolute cell count was determined from samples of peripheral blood as previously described (46). Briefly, $50 \mu \mathrm{l}$ of whole blood was placed in a polypropylene tube and incubated with fluorescein isothiocyanate-conjugated anti-CD3, phycoerythrin-conjugated anti-CD4 (Leu-3a; Becton Dickinson), and peridinin chlorophyll protein-conjugated anti-CD8 at $4^{\circ} \mathrm{C}$. Residual red blood cells were removed with FACS lysing solution (Becton Dickinson), and the cells were analyzed on a FACSCalibur (Becton Dickinson) by using CellQuest software (Becton Dickinson).

Plasma viral RNA copy number. Plasma viral RNA copy numbers were measured by a real-time quantification assay based on the Applied Biosystems Prism 7700 sequence detection system (PE Biosystems, Foster City, Calif.) (34). Viral RNA was isolated from plasma by using a QIAamp Viral RNA Mini kit (Qiagen, Valencia, Calif.). The RNA was subjected to reverse transcription-PCR (with recombinant Tth DNA polymerase, SIV Gag consensus primers (SIVmac2391224F and SIVmac239-1326R), and the SIV Gag consensus Taqman probe, FAM-SIV-1272T. SIV Gag DNA-PCR was carried out as previously described (37).

Statistical analysis. Data are expressed as the mean \pm the SD, and data analysis was carried out by using the StatView program (SAS Institute, Cary, N.C.). A $P$ value of $<0.05$ was considered significant.

\section{RESULTS}

Construction of recombinant vaccinia virus DIs expressing SIV Gag. To study the ability of an rDI-based vaccine to induce protective immunity, the full-length gag gene of SIVmac239 was selected for vector construction. rDIs expressing SIVmac239 Gag (rDIsSIVGag) and a control vector expressing the gene for LacZ (rDIsLacZ) were constructed, and the purified virions were used to infect CEF cells (Fig. 1). A 55$\mathrm{kDa}$ band corresponding to SIV Gag was detected by Western blot in extracts from CEF cells infected with rDIsSIVGag by using anti-SIV Gag-specific MAbs (IB6 or V10) (Fig. 1). The presence of DNA encoding SIV Gag in virions was confirmed by DNA PCR (data not shown). In contrast, Gag proteins and DNA were not detected in CEF cells infected with rDIsLacZ.

Detection of intracellular SIV p27 in mammalian cells infected with rDIsSIVGag. Mammalian cell lines, such as BHK21, RK13, and CHO, were shown to variously support replication of MVA, as previously reported by Carroll et al. (7). By 
$\mathbf{a}$

VV-DIs

VV-DIsLacZ

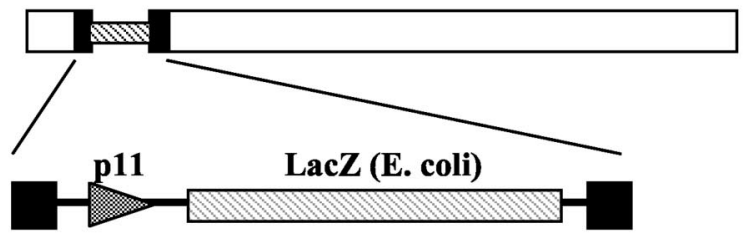

VV-DIsSIVGag
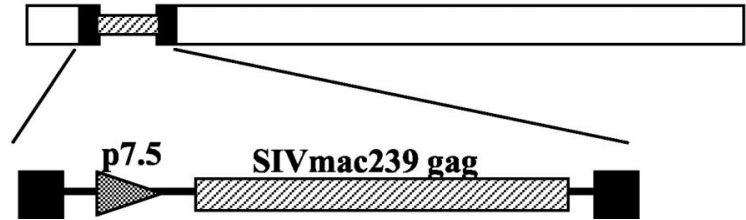

b

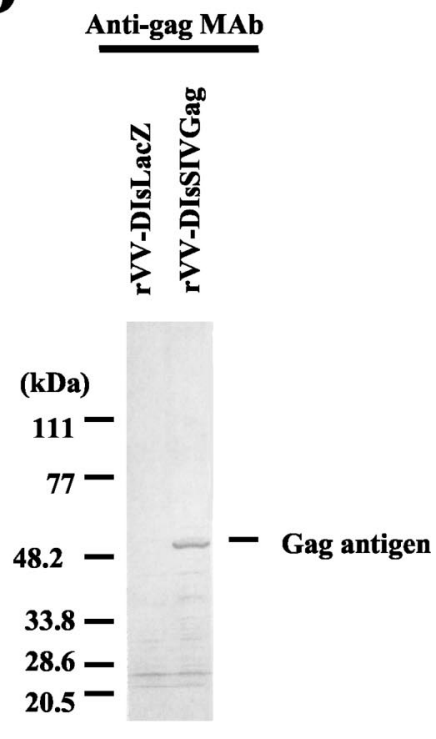

FIG. 1. Vector construction and expression of rDIsSIVGag. (a) Construction of rDIsSIVGag. Full-length DNA of SIVmac239 Gag was inserted into the deleted region of vaccinia strain DIs. (b) Detection of SIV Gag protein by Western blot with anti-p27 Gag MAb IB6.

comparison, exposure of mammalian cells to rDIsSIVGag did not yield infectious virus, although p27 antigen was expressed. In CEF cells, high levels of SIV p27 antigen were detected intracellularly with $15.8 \pm 3.7 \mathrm{ng}$ of protein $/ 10^{6}$ cells after exposure to rDIsSIVGag (Table 1). In contrast, rDIsSIVGag was completely replication defective in mammalian cell lines, such as BHK-21, RK13, and CHO (Table 1). However, the levels of p27 antigen production were significant in each of the mammalian cells at $1,375,275$, and $119 \mathrm{pg} / \mathrm{ml}$ compared to controls (Table 1). Thus, the p27 antigen production between CEF and mammalian cells by rDIsSIVGag exposure was significantly different, and its reduction rates of BHK-21, RK13, and CHO to CEF cells were $8.7,1.74$, and $0.75 \%$, respectively, presumably reflecting differences in the ability of the virus to replicate in these cells. By comparison, wild-type recombinant vaccinia rWRSIVGag produced high levels of p27 in infected cells, whereas p27 was not detected after exposure to rDIsLacZ.

Immunization of rDIsSIVGag and immune induction. Based on the expression of SIV p27 in mammalian cells, the ability of rDIsSIVGag to induce virus-specific immunity was investigated by using a SHIV/macaque model (Table 2). A total of eight cynomologus macaques were evaluated. Three macaques were intravenously inoculated with rDIsSIVGag $\left(10^{6}\right.$ PFU) and boosted at 24 weeks postinoculation (p.i.) with the same dose of antigen. Three other macaques were inoculated with rDIsLacZ $\left(10^{6} \mathrm{PFU}\right)$ as a vector control. The two remaining macaques served as naive controls. At 6 weeks after the booster inoculation, the macaques were intravenously challenged with 20 TCID $_{50}$ of highly pathogenic SHIV-C2/1, which was obtained by serum passage of SHIV-89.6 (37). The effects of vaccination with rDIsSIVGag on immune induction were monitored for 12 weeks, and the macaques were then autopsied.

Antigen-specific T-cell responses in all eight macaques were
TABLE 1. Replication of rDIsSIVGag and other vaccinia virus recombinants and production of SIV Gag p27 protein $^{a}$

\begin{tabular}{lccc}
\hline \multirow{2}{*}{$\begin{array}{c}\text { Cell type and } \\
\text { vaccinia virus }\end{array}$} & $\begin{array}{c}\text { Mean amt of } \\
\text { virus } \\
\text { replication } \\
\text { SD }\end{array}$ & \multicolumn{2}{c}{$\begin{array}{c}\text { Mean p27 antigen amt }(\mathrm{pg} / \mathrm{ml}) \pm \mathrm{SD} \\
\text { in } 10^{6} \text { cells }\end{array}$} \\
\cline { 3 - 4 } & & Supernatant & Cell lysate \\
\hline $\begin{array}{l}\text { CEF cells } \\
\text { rWRSIVGag }\end{array}$ & $9,500 \pm 1,800$ & $6,530 \pm 2,600$ & $87,300 \pm 14,800$ \\
rDIsSIVGag & $1,860 \pm 350$ & $2,470 \pm 60$ & $15,800 \pm 3,700$ \\
rDIsLacZ & $1,400 \pm 240$ & $<20$ & $<20$ \\
MVA & $10,327 \pm 1,479$ & $<20$ & $<20$ \\
& & & \\
BHK-21 cells & & & \\
rWRSIVGag & $2,500 \pm 400$ & $970 \pm 22$ & $34,600 \pm 12,700$ \\
rDIsSIVGag & $<1$ & $<20$ & $1,375 \pm 273$ \\
rDIsLacZ & $<1$ & $<20$ & $<20$ \\
MVA & $2,250 \pm 835$ & $<20$ & $<20$ \\
& & & \\
RK13 cells & & & $<20$ \\
rWRSIVGag & $1,500 \pm 300$ & $350 \pm 12$ & $16,300 \pm 9,270$ \\
rDIsSIVGag & $<1$ & $<20$ & $275 \pm 34$ \\
rDIsLacZ & $<1$ & $<20$ & $<20$ \\
MVA & $125 \pm 102$ & $<20$ & $<20$ \\
CHO cells & & & $<20$ \\
rWRSIVGag & $953 \pm 347$ & $410 \pm 120$ & $5,320 \pm 7,670$ \\
rDIsSIVGag & $<1$ & $<20$ & $119 \pm 34$ \\
rDIsLacZ & $<1$ & $<20$ & $<20$ \\
MVA & $92 \pm 49$ & $<20$ & $<20$ \\
\hline
\end{tabular}

${ }^{a} \mathrm{CEF}, \mathrm{RK} 13$, and BHK-21 cells were infected with rDIs or rWR strain viruses at an MOI of 0.05 for the detection of virus replication and at an MOI of 1.0 for p27 antigen generation in the culture supernatant and cells. p27 antigen was measured by enzyme-linked immunosorbent assay in cultures containing $10^{6}$ cells $/ \mathrm{ml}$, and the data are presented as the average value \pm the SD.

${ }^{b}$ For analysis of virus replication, mammalian cells were infected with each virus at an MOI of 0.05 . The cells were harvested at 0 and $48 \mathrm{~h}$ after adsorption and sonicated. Virus replication was determined by dividing the virus yield at $48 \mathrm{~h}$ by that at $0 \mathrm{~h} \mathrm{(15)}$. Values of $<1$ and $<20 \mathrm{pg} / \mathrm{ml}$ represent the detection limits of the virus replication and p27 antigen assays, respectively. 
TABLE 2. Immunization and challenge schedule ${ }^{a}$

\begin{tabular}{|c|c|c|c|}
\hline Group & Macaque(s) & Immunization and schedule & Challenge \\
\hline 1 & C623, C975, and C977 & rDIs (rDIsSIVGag), $10^{6}$ PFU, i.v., wk 0 and 24 & SHIV-C2/1, 20-TCID ${ }_{50}$, i.v., wk 30 \\
\hline 2 & C971, C973, and C978 & Control DIs (rDIsLacZ), $10^{6}$ PFU, i.v., wk 0 and 24 & SHIV-C2/1, 20 TCID $_{50}$, i.v., wk 30 \\
\hline 3 & C699 and C972 & Naive, PBS, i.v., wk 0 and 24 & SHIV-C2/1, 20 TCID $_{50}$, i.v., wk 30 \\
\hline
\end{tabular}

${ }^{a}$ Immunization and virus challenge were conducted simultaneously for all groups. The times of immunization and challenge were indicated as weeks after initial immunization. i.v., intravenous inoculation.

monitored by IFN- $\gamma$-ELISPOT assays. IFN- $\gamma$-specific SFC were detected after the initial immunization with rDIsSIVGag (101 $\pm 14 \mathrm{SFC} / 10^{6} \mathrm{PBMC}$ at 2 weeks p.i.) and increased significantly by 2 weeks after the booster immunization ( $603 \pm$ $23 \mathrm{SFC} / 10^{6} \mathrm{PBMC}$ at 26 weeks p.i.) and by 1 week after SHIV challenge (650 $\pm 42 \mathrm{SFC} / 10^{6} \mathrm{PBMC}$ at 31 weeks p.i.) (Fig. 2). In contrast, the number of SFC in controls, including naive macaques, were significantly lower (fewer than 20 SFC before challenge and fewer than 100 SFC after challenge). Thus, intravenous inoculation of rDIsSIVGag induced significant $\mathrm{T}$ cell responses specific for SIV p27 Gag in the immunized macaques.

In vitro lymphocyte proliferative responses were also evaluated by using macaque PBMC isolated 2 weeks after the booster inoculation (Fig. 3). Macaques immunized with rDIsSIVGag showed high levels of proliferative responses specific for purified, native $\mathrm{p} 27$ protein, with a mean stimulation index of $24.77 \pm 4.1$. No significant responses were detected in macaques inoculated with the control vector $(P<0.001)$. Proliferative responses to ConA, used as a positive control, were significantly higher in all macaques, with a mean stimulation index of $148.67 \pm 10.7$. These results show that rDIsSIVGag can induce high levels of T-lymphocyte proliferative responses in immunized macaques. Taken together, these findings suggest that vaccination with rDIsSIVGag induces sufficient virus-specific immunity to warrant studying the efficacy of these responses against pathogenic SHIV challenge.

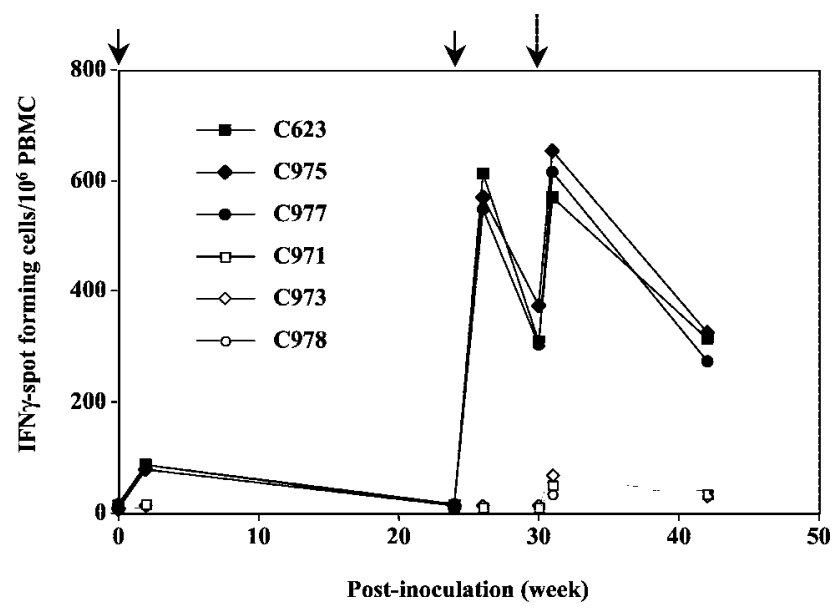

FIG. 2. SIV Gag-specific IFN- $\gamma$ SFC in PBMC from rDIsSIVGagand rDIsLacZ-inoculated and naive macaques. Freshly isolated PBMC were assessed for their ability to produce IFN- $\gamma$ in response to overlapping peptides covering the full-length SIV Gag protein. The experimental schedule of each grouped animal is presented in Table 2.
SHIV challenge enhances virus-specific intracellular IFN$\gamma$-staining and anti-Gag antibody responses in rDIsSIVGagvaccinated macaques. To evaluate the induction of immune responses before and after SHIV challenge, PBMC from immunized macaques were stained for surface CD8 and intracellular IFN- $\gamma$ expression, followed by flow cytometric analyses. Macaques immunized with rDIsSIVGag generated $0.61 \% \pm$ $0.14 \% \mathrm{CD}^{+} \mathrm{IFN}-\gamma^{+}$double-positive cells at week 26 p.i. or at 2 weeks after the second immunization of rDIs, recombinant, whereas the vector control animals had less than $0.02 \%$ double-positive cells (Fig. 4a). At week 31 p.i. or 1 week postSHIV-challenge, the percentage of $\mathrm{CD}^{+}{ }^{+}$IFN- $\gamma^{+}$double-positive cells had increased to $1.25 \% \pm 0.09 \%$ in the rDIsSIVGagimmunized macaques $(P<0.05)$, whereas vector controls showed fewer than $0.02 \%$ double-positive cells (Fig. 4b). As shown in Fig. 2 and 4, enhancement of numbers in both IFN$\gamma$-specific intracellular staining (ICS)-positive cells and ELISPOTs by SHIV challenge were evident in rDIsSIVGagimmunized animals.

The effect of SHIV challenge on anti-p27 Gag antibody titers was also evaluated in the sera of immunized macaques (Fig. 5). After the initial inoculation and boost with rDIsSIVGag, all of the macaques produced significant levels of anti-p27 antibodies, with a mean titer of 3,041 $\pm 1,612$ at the time of SHIV challenge. Antibody titers rapidly increased to a mean of 7,160 $\pm 4,227$ by 2 weeks postchallenge $(P<0.005)$. In contrast, SIV-specific antibodies were not detected in macaques inoculated with the control vector, nor were they detected in naive control animals during the early phase of viral infection.

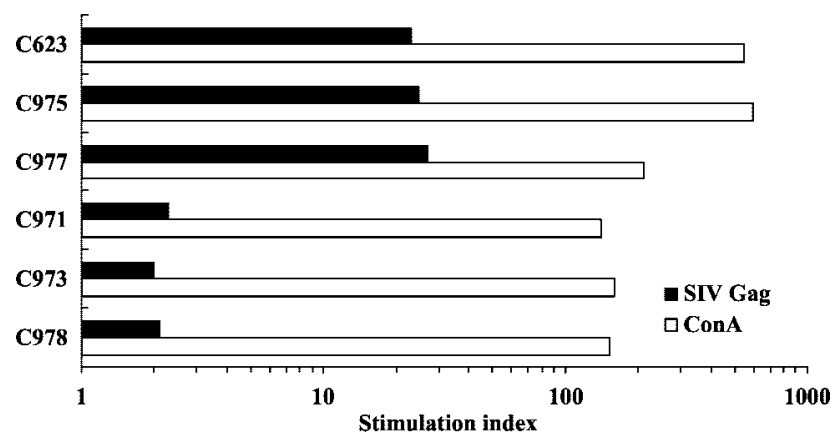

FIG. 3. Proliferative responses to SIV p27 Gag in the macaques inoculated with either rDIsSIVGag or rDIsLacZ. Responses were measured 2 weeks after the second inoculation of recombinant vaccinia virus antigen. Naive macaques did not show any proliferative response to SIV p27 (data not shown). Bars represent the mean values of all animals. 
a 26 week (at 2 week post-2nd immunization) $\mathbf{b}$ 31 week (at 1 week post-SHIV challenge)

$\mathbf{C 6 2 3}$
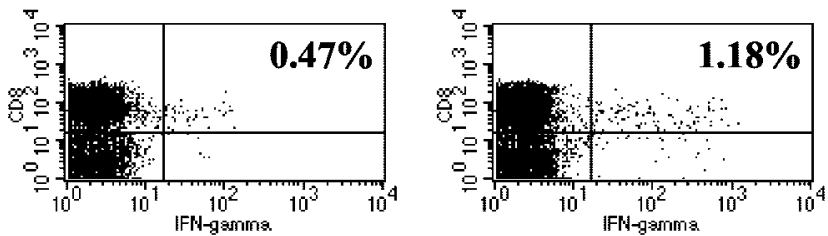

C975
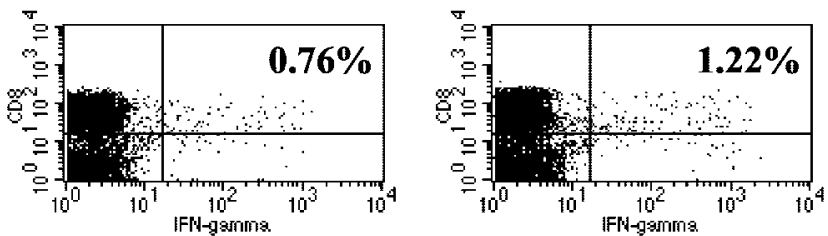

C977
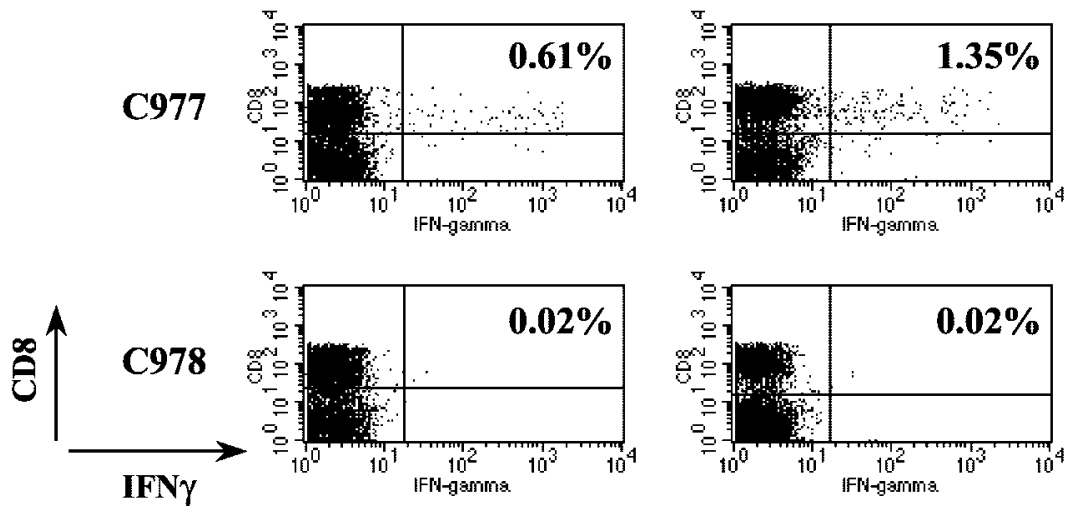

FIG. 4. Flow cytometric analysis of IFN- $\gamma$-producing $C D 8^{+} \mathrm{T}$ cells specific for SIV Gag. PBMC from macaques were cultured in vitro with overlapping peptides and stained for intracellular IFN- $\gamma$. The percentage of IFN- $\gamma$-producing $\mathrm{CD}^{+} \mathrm{T}$ cells in each macaque PBMC was determined by flow cytometry, and data obtained before (at 26 week p.i. [a]) and after (at 31 week p.i. [b]) the SHIV challenge were compared.

CD4 ${ }^{+}$-T-cell counts and plasma viral load after SHIV challenge are controlled in rDIsSIVGag-vaccinated macaques. All eight macaques were challenged intravenously with $20 \mathrm{TCID}_{50}$ of a pathogenic SHIV at 30 weeks p.i. (Table 2). The naive macaques and vector control animals developed a rapid and complete loss of $\mathrm{CD}^{+} \mathrm{T}$ lymphocytes and intense primary viremia, with peak values reaching a mean of $4.27 \times 10^{8}$ RNA copies/ml during the first 10 to 14 days postchallenge. Viral

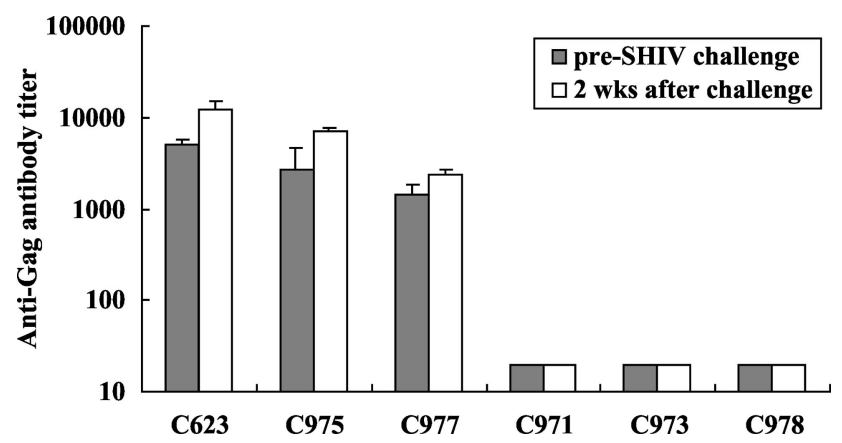

FIG. 5. Enhancement of anti-SIV Gag antibody titers in rDIsSIVGag-inoculated macaques by SHIV challenge. Titers of binding antibody in plasma to SIV Gag were measured by SIV Gag-specific enzyme-linked immunosorbent assay. The results represent the mean of three independent experiments. Error bars represent the mean \pm three SD. set-point levels of $5 \times 10^{6}$ to $5 \times 10^{7} \mathrm{RNA}$ copies $/ \mathrm{ml}$ were maintained during the acute phase of infection (Fig. 6). In contrast, all three macaques immunized with rDIsSIVGag showed almost stable $\mathrm{CD}^{+}{ }^{+}$-T-lymphocyte counts and significantly reduced the set-point levels, with plasma viral RNA copy numbers 40 - to 200 -fold lower than those of vector controls.

The reduction in plasma viral load and inhibition of $\mathrm{CD} 4^{+}$ $\mathrm{T}$-cell loss were reflected in the maintenance of $\mathrm{T}$ lymphocytes and lymphoid architecture in the spleen and mesenteric lymph nodes in rDIsSIVGag-vaccinated animals (Fig. 7a and c, respectively). In contrast, spleen and mesenteric lymph nodes in vector controls and naive macaques showed that lymphoid cells were significantly reduced and architecture was disrupted and lost (Fig. 7b and d, respectively). CD4 ${ }^{+} \mathrm{T}$ lymphocytes isolated from fresh spleen tissues and lymph nodes from the autopsied macaques were evaluated by flow cytometry. $\mathrm{CD}^{+} \mathrm{T}$ cells were significantly maintained in various lymphoid tissues from macaques immunized with rDIsSIVGag, with mean values of $19.9 \% \pm 6.0 \%, 24.9 \% \pm 12.4 \%$, and $20.6 \% \pm 13.1 \%$ in spleen, inguinal lymph nodes, and mesenteric lymph nodes, respectively (Fig. 7e). Compared to values derived from normal, healthy cynomologus macaques (37), the mean relative percentages of $\mathrm{CD}^{+}{ }^{+} \mathrm{T}$ cells remaining in the spleen, inguinal lymph nodes, and mesenteric lymph nodes were $21 \% \pm 15 \%$, $49 \% \pm 12 \%$, and $46 \% \pm 12 \%$, respectively. These results show 

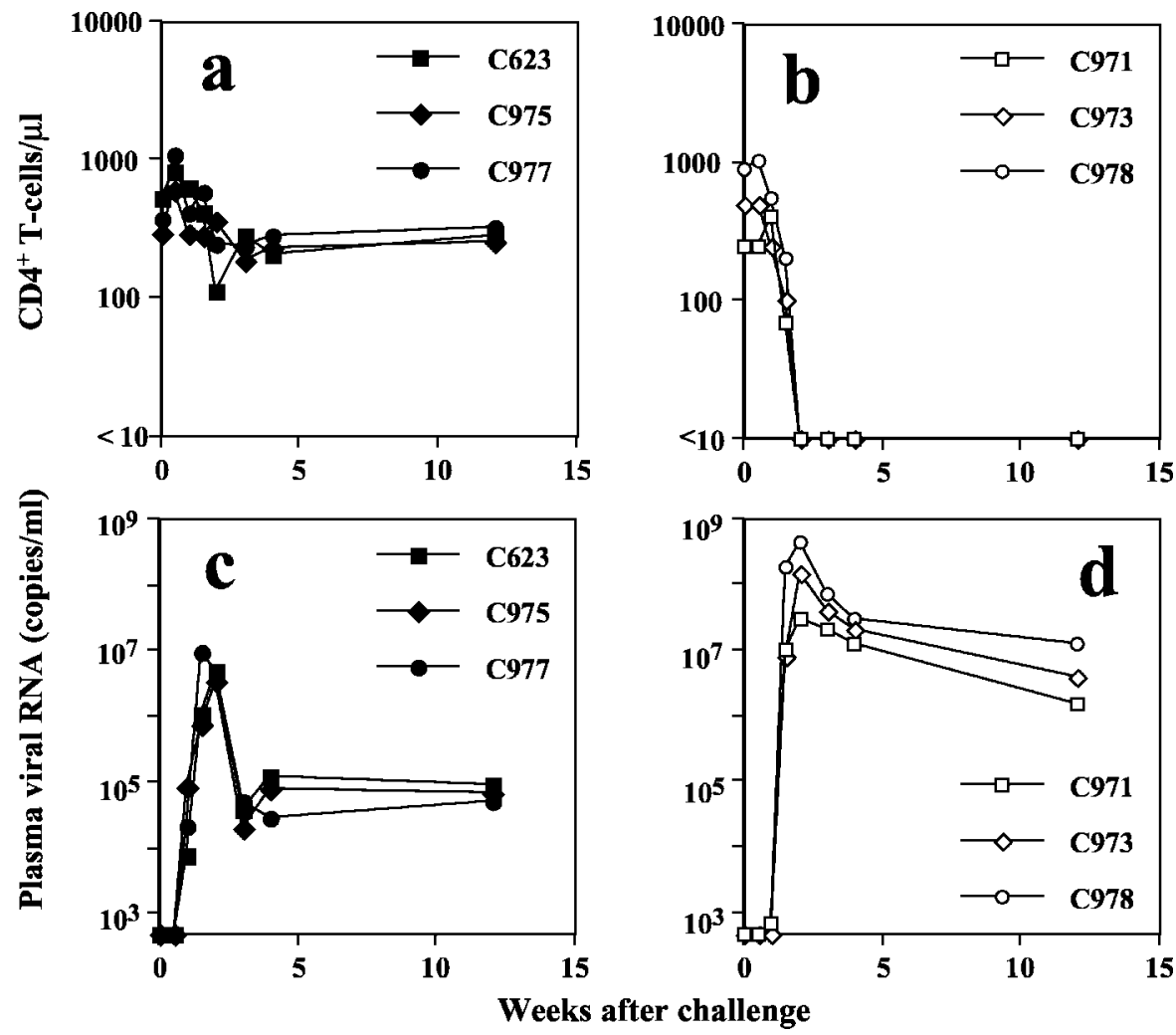

FIG. 6. Comparison of $\mathrm{CD}^{+} \mathrm{T}$ cells and viral load in plasma among rDIsSIVGag-inoculated macaques and vector controls. (a and b) CD4 ${ }^{+}$-T-cell counts in peripheral blood of rDIsSIVGag-inoculated animals (a) and vector controls (b). (c and d) Viral set-point levels in plasma in vaccinated macaques (c) and vector controls (d).

that nearly half of the relative percentages of tissue $\mathrm{CD} 4^{+} \mathrm{T}$ cells were maintained during the acute phase of a highly pathogenic SHIV infection as a result of vaccination with rDIsSIVGag. In contrast, tissue $\mathrm{CD} 4^{+}-\mathrm{T}$-cell numbers were reduced to $<1.0 \%$ in the control macaques (Fig. 7e).

\section{DISCUSSION}

We have previously demonstrated that the attenuated vaccinia virus strain DIs is completely replication deficient in various mammalian cell lines in vitro $(17,25,41)$, making it an attractive candidate for HIV-1 vector-based vaccine development (15). rDIs expressing HIV-1 clade B Gag induced high levels of T-cell responses in mice (15). In preliminary studies, DIs was found to be safe for use in humans based on a clinical trial to examine its utility as a smallpox vaccine ( $\mathrm{K}$. Matsuo et al., unpublished results). However, the ability of rDIs to provide protective immunity in the SIV/macaque model is unknown.

In the present study, we produced rDIsSIVGag expressing SIV Gag and found that both DIs and rDIs were replication deficient in mammalian cells. We examined the replicative abilities of these viruses in both CEF and mammalian cells in comparison to MVA. Our results demonstrate that MVA replicates well in CEF and BHK-21 cells and in other mammalian cells, such as RK13 and CHO cells. These results are similar to those of a previous report (6). In contrast, DIs and rDIs replicated only in CEF but not in the other mammalian cells described in Table 1. Interestingly, rDIsSIVGag produced sig- nificant amounts of SIV Gag protein in all of the mammalian cells tested, although the production levels were decreased from ca. 9 to $0.8 \%$ in mammalian cells compared to CEF. The discrepancy between virus replication and protein generation in rDIsSIVGag-infected cells may depend on the deletion of genes, including a 15.4-kbp fragment from regions $\mathrm{C}$ to $\mathrm{K}$ of the vaccinia virus genome (15). This fragment includes some known functional genes, such as host range genes and the late-phase gene involved in viral protein production. Although the deleted region in DIs is larger than that of MVA, the precise mechanism that results in the discrepancy between virus replication and the generation of viral protein is still not fully understood.

The goal of the present study was to determine whether a vaccine consisting of rDIs expressing full-length SIV Gag (rDIsSIVGag) could induce protection in macaques against a highly pathogenic SHIV challenge. Intravenous inoculation of rDIsSIVGag in cynomologus macaques resulted in significant control of plasma viremia and maintenance of $\mathrm{CD}^{+} \mathrm{T}$ cells during acute primary infection. Immunization with rDIsSIVGag also minimized the loss of tissue $\mathrm{CD}^{+}{ }^{+} \mathrm{T}$ cells in various lymphoid organs, including spleen and lymph nodes. This was associated with high levels of antigen-specific proliferative responses and an increase in the number of IFN- $\gamma$ SFC in the vaccinated animals. Furthermore, virus-specific, IFN- $\gamma$-specific ICS and antibody responses were significantly enhanced by pathogenic SHIV challenge in the macaques. 

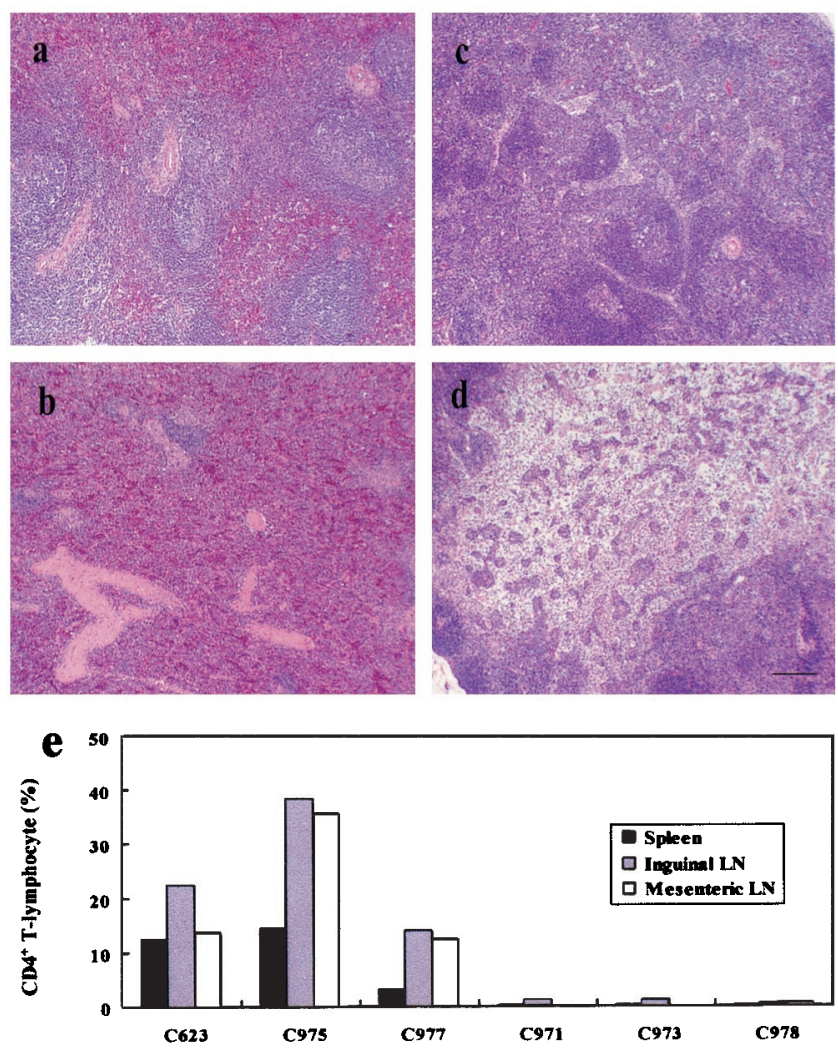

FIG. 7. $\mathrm{CD}^{+} \mathrm{T}$ lymphocytes remaining in spleen and mesenteric lymph nodes of macaques inoculated with rDIsSIVGag. Morphological analysis of spleen and mesenteric lymph node from vaccinated macaques. Tissue sections of spleen ( $\mathrm{a}$ and $\mathrm{b}$ ) and lymph nodes ( $\mathrm{c}$ and d) were obtained at autopsy from macaques inoculated with rDIsSIVGag (a and c) or rDIsLacZ (b and d), followed by SHIV challenge. Sections were obtained from samples taken 12 weeks postchallenge and were stained with hematoxylin and eosin. Bar, $100 \mu \mathrm{m}$. (e) Quantitative flow cytometric analysis of $\mathrm{CD} 4^{+} \mathrm{T}$ lymphocytes in the spleen, inguinal lymph nodes, and mesenteric lymph nodes. The data represent mean values from the spleen and three different tissues of each inguinal and mesenteric lymph node.

The present study directly addressed the ability of an rDIsbased vaccine to afford protection from pathogenic virus challenge. We found that the relative content of tissue $\mathrm{CD}^{+}{ }^{+} \mathrm{T}$ cells was reduced to nearly half the normal values in spleen and lymph nodes in the rDIsSIVGag-vaccinated macaques after SHIV challenge. However, since absolute numbers of total lymphoid cells increased from 4- to 10-fold in the tissues of the vaccinated macaques, the absolute number of $\mathrm{CD}^{+} \mathrm{T}$ cells was estimated to increase 2- to 5-fold in the lymphoid tissues. Similar increases were not observed in macaques in the control groups. This may be associated with the maintenance of circulating $\mathrm{CD}^{+}{ }^{+} \mathrm{T}$ cells in the immunized macaques after SHIV challenge. Indeed, the number of virus-specific IFN- $\gamma$-specific ICS and anti-Gag antibody titers were significantly enhanced by SHIV challenge in the vaccinated group, suggesting that immune function was maintained. Thus, although the reduction in plasma viremia was only partial, virus-specific immune enhancement after SHIV challenge and maintenance of $\mathrm{CD}^{+}$ $\mathrm{T}$ cells were significant in macaques intravenously vaccinated with rDIsSIVGag.
Although it is not possible to fully compare protective efficacy among different vaccine models, our results appear to be similar to those obtained with recombinant MVA expressing multiple SIV proteins encoded by env, gag, and pol (3, 4, 30, 35, 36). Therefore, rDIsSIVGag, which expresses a single SIV protein, is perhaps similarly effective at inducing protection of macaques from pathogenic SHIV infection. MVA and DIs are derived from different vaccinia virus strains, but both are highly attenuated, and the immunogenicity seems to be similar between the two.

One important factor contributing to our results is the use of an intravenous route for vaccine administration. DIs and its vectorbased recombinants have an advantage when administered intravenously, because neither can replicate in any mammalian cell lines tested thus far. It is the "nonreplicative" character of this vaccine that may be especially beneficial at inducing immunity to foreign antigens when relatively large amounts of the vaccine are administered intravenously. By comparison, the intravenous administration of a low-replicative vaccine strain, BCG, induced antigen-nonspecific hyperactivation of lymphoid cells $(16,48)$. Thus, intravenous inoculation of rDIs at a dose of $10^{6}$ PFU might result in effective immune induction without inducing a hyperinflammatory reaction.

Although the present study did not directly address the effect of different administration routes for immune induction, it is evident that intravenous inoculation is not practical for use in humans. In a preliminary study by our group to determine the efficacy of different routes of vaccination with long-term follow-up, inoculation of $10^{6}$ or $10^{7}$ PFU of rDIsSIVGag by an intradermal route induced similar levels of antigen-specific immunity and protection against SHIV challenge in cynomolgus macaques (Y. Izumi et al., unpublished data), suggesting the possibility of administration to humans.

It is probable that the control of plasma viral load and $\mathrm{CD}^{+}{ }^{-}$-T-cell counts in PBMC and other general lymphoid tissues after SHIV challenge was mediated by virus-specific cellular immune responses. The number of Gag-specific IFN- $\gamma$ SFC in PBMC peaked around 2 weeks after the booster inoculation in all of the vaccinated macaques. At the same time, proliferative responses to SIV p27 reached relatively high levels in the same animals. Although some controversy exists between the association of proliferative responses with protection against pathogenic SHIV infection $(13,39)$, our results are partially consistent with those of Gauduin et al., which indicate that SIV-specific T-lymphocyte responses induced by immunization with a live attenuated SIV vaccine play a role in protective immunity (11). Notably, both virus-specific IFN- $\gamma$-specific ICS-positive $\mathrm{CD}^{+} \mathrm{T}$ cells and anti-Gag antibody responses were strikingly enhanced by challenge with pathogenic SHIV. Although the vaccine target was only SIV Gag in this strategy, anti-Gag antibody titers were significantly higher than those induced in macaques immunized with MVA or DNA/MVA expressing Gag-Pol-Env $(2,3)$. This could account for the higher inducible ability of virus-specific proliferative responses or helper cell responses in rDIsSIVGag-immunized animals, which might be associated with the enhancement of antibody production not only against SIV Gag but also against SHIV Env protein. It may therefore be reasonable to speculate that anti-SHIV Env antibodies or even neutralizing antibody might be effectively induced in the vaccinated macaques after challenge. It is possible that protection achieved by immuni- 
zation with rDIsSIVGag was due not only to cellular immune responses but also to the induction of humoral immunity in the vaccinated macaques. In summary, the present study revealed that vaccination of cynomologus macaques with a completely replication-deficient vaccinia virus DIsSIVGag recombinant resulted in significant control of viral load and $\mathrm{CD}^{+}{ }^{+}$-T-cell maintenance after challenge with pathogenic SHIV.

\section{ACKNOWLEDGMENTS}

We thank Bernard Moss, Laboratory of Viral Diseases, National Institute of Allergy and Infectious Diseases, National Institutes of Health, Bethesda, Md., for helpful discussions. We also thank Hidemi Takahashi and Yohko Nakagawa, Department of Microbiology and Immunology, Nippon Medical School, Tokyo, Japan, for helpful discussions.

The "Panel on AIDS" of the U.S.-Japan Cooperative Medical Science Program, the Human Science Foundation of Japan, and the Japanese Ministry of Health, Labor, and Welfare supported this work. This study was also supported by the AIDS Vaccine Project in conjunction with the Japan Science and Technology Corporation.

\section{REFERENCES}

1. Allen, T. M., J. Sidney, M. F. del Guercio, R. L. Glickman, G. L. Lensmeyer, D. A. Wiebe, R. DeMars, C. D. Pauza, R. P. Johnson, A. Sette, and D. I. Watkins. 1998. Characterization of the peptide binding motif of a rhesus MHC class I molecule (Mamu-A*01) that binds an immunodominant CTL epitope from simian immunodeficiency virus. J. Immunol. 160:6062-6071.

2. Amara, R. R., F. Villinger, J. D. Altman, S. L. Lydy, S. P. O'Neil, S. I. Staprans, D. C. Montefiori, Y. Xu, J. G. Herndon, L. S. Wyatt, M. A Candido, N. L. Kozyr, P. L. Earl, J. M. Smith, H. L. Ma, B. D. Grimm, M. L. Hulsey, J. Miller, H. M. McClure, J. M. McNicholl, B. Moss, and H. L. Robinson. 2001. Control of a mucosal challenge and prevention of AIDS by a multiprotein DNA/MVA vaccine. Science 292:69-74.

3. Amara, R. R., F. Villinger, S. I. Staprans, J. D. Altman, D. C. Montefiori, N. L. Kozyr, Y. Xu, L. S. Wyatt, P. L. Earl, J. G. Herndon, H. M. McClure, B. Moss, and H. L. Robinson. 2002. Different patterns of immune responses but similar control of a simian-human immunodeficiency virus $89.6 \mathrm{P}$ mucosa challenge by modified vaccinia virus Ankara (MVA) and DNA/MVA vaccines. J. Virol. 76:7625-7631.

4. Barouch, D. H., S. Santra, M. J. Kuroda, J. E. Schmitz, R. Plishka, A Buckler-White, A. E. Gaitan, R. Zin, J. H. Nam, L. S. Wyatt, M. A. Lifton, C. E. Nickerson, B. Moss, D. C. Montefiori, V. M. Hirsch, and N. L. Letvin. 2001. Reduction of simian-human immunodeficiency virus 89.6P viremia in rhesus monkeys by recombinant modified vaccinia virus Ankara vaccination. J. Virol. 75:5151-5158.

5. Barouch, D. H. S. Santra, J. E. Schmitz, M. J. Kuroda, T. M. Fu, W. Wagner, M. Bilska, A. Craiu, X. X. Zheng, G. R. Krivulka, K. Beaudry, M. A. Lifton, C. E. Nickerson, W. L. Trigona, K. Punt, D. C. Freed, L. Guan, S Dubey, D. Casimiro, A. Simon, M. E. Davies, M. Chastain, T. B. Strom, R. S. Gelman, D. C. Montefiori, M. G. Lewis, E. A. Emini, J. W. Shiver, and N. L. Letvin. 2000. Control of viremia and prevention of clinical AIDS in rhesus monkeys by cytokine-augmented DNA vaccination. Science 290:486-492.

6. Carroll, M. W., and B. Moss. 1997. Host range and cytopathogenicity of the highly attenuated MVA strain of vaccinia virus: propagation and generation of recombinant viruses in a nonhuman mammalian cell line. Virology 238:198-211.

7. Carroll, M. W., W. W. Overwijk, R. S. Chamberlain, S. A. Rosenberg, B. Moss, and N. P. Restifo. 1997. Highly attenuated modified vaccinia virus Ankara (MVA) as an effective recombinant vector: a murine tumor model Vaccine 15:387-394.

8. Egan, M. A., W. A. Charini, M. J. Kuroda, J. E. Schmitz, P. Racz, K. Tenner-Racz, K. Manson, M. Wyand, M. A. Lifton, C. E. Nickerson, T. Fu, J. W. Shiver, and N. L. Letvin. 2000. Simian immunodeficiency virus (SIV) gag DNA-vaccinated rhesus monkeys develop secondary cytotoxic T-lymphocyte responses and control viral replication after pathogenic SIV infection. J. Virol. 74:7485-7495.

9. Evans, D. T., D. H. O’Connor, P. Jing, J. L. Dzuris, J. Sidney, J. da Silva, T. M. Allen, H. Horton, J. E. Venham, R. A. Rudersdorf, T. Vogel, C. D. Pauza, R. E. Bontrop, R. DeMars, A. Sette, A. L. Hughes, and D. I. Watkins. 1999. Virusspecific cytotoxic T-lymphocyte responses select for amino-acid variation in simian immunodeficiency virus Env and Nef. Nat. Med. 5:1270-1276.

10. Fauci, A. S., S. M. Schnittman, G. Poli, S. Koenig, and G. Pantaleo. 1991 NIH conference. Immunopathogenic mechanisms in human immunodeficiency virus (HIV) infection. Ann. Intern. Med. 114:678-693.

11. Gauduin, M. C., R. L. Glickman, S. Ahmad, T. Yilma, and R. P. Johnson. 1999. Characterization of SIV-specific $\mathrm{CD}^{+}{ }^{+}$T-helper proliferative responses in macaques immunized with live-attenuated SIV. J. Med. Primatol. 28:233-241.
12. Hanke, T., R. V. Samuel, T. J. Blanchard, V. C. Neumann, T. M. Allen, J. E. Boyson, S. A. Sharpe, N. Cook, G. L. Smith, D. I. Watkins, M. P. Cranage, and A. J. McMichael. 1999. Effective induction of simian immunodeficiency virus-specific cytotoxic $\mathrm{T}$ lymphocytes in macaques by using a multiepitope gene and DNA prime-modified vaccinia virus Ankara boost vaccination regimen. J. Virol. 73:7524-7532.

13. Heeney, J. L., V. J. Teeuwsen, M. van Gils, W. M. Bogers, C. De Giuli Morghen, A. Radaelli, S. Barnett, B. Morein, L. Akerblom, Y. Wang, T. Lehner, and D. Davis. 1998. $\beta$-Chemokines and neutralizing antibody titer correlate with sterilizing immunity generated in HIV-1 vaccinated macaques. Proc. Natl. Acad. Sci. USA 95:10803-10808.

14. Hel, Z., D. Venzon, M. Poudyal, W. P. Tsai, L. Giuliani, R. Woodward, C. Chougnet, G. Shearer, J. D. Altman, D. Watkins, N. Bischofberger, A Abimiku, P. Markham, J. Tartaglia, and G. Franchini. 2000. Viremia control following antiretroviral treatment and therapeutic immunization during primary SIV251 infection of macaques. Nat. Med. 6:1140-1146.

15. Ishii, K., Y. Ueda, K. Matsuo, Y. Matsuura, T. Kitamura, K. Kato, Y. Izumi, K. Someya, T. Ohsu, M. Honda, and T. Miyamura. 2002. Structural analysis of vaccinia virus DIs strain: application as a new replication-deficient viral vector. Virology 302:433-444.

16. Kawahara, M., T. Nakasone, and M. Honda. 2002. Dynamics of gamma interferon, interleukin-12 (IL-12), IL-10, and transforming growth factor $\beta$ mRNA expression in primary Mycobacterium bovis BCG infection in guinea pigs measured by a real-time fluorogenic reverse transcription-PCR assay. Infect. Immun. 70:6614-6620.

17. Kitamura, T., Y. Kitamura, and I. Tagaya. 1967. Immunogenicity of an attenuated strain of vaccinia virus on rabbits and monkeys. Nature 215:1187-1188.

18. Lehner, T., and G. M. Shearer. 2002. Alternative HIV vaccine strategies. Science 297:1276-1277.

19. Lehner, T., Y. Wang, M. Cranage, L. A. Bergmeier, E. Mitchell, L. Tao, G Hall, M. Dennis, N. Cook, R. Brookes, L. Klavinskis, I. Jones, C. Doyle, and R. Ward. 1996. Protective mucosal immunity elicited by targeted iliac lymph node immunization with a subunit SIV envelope and core vaccine in macaques. Nat. Med. 2:767-775.

20. Lu, Y., M. S. Salvato, C. D. Pauza, J. Li, J. Sodroski, K. Manson, M. Wyand, N. Letvin, S. Jenkins, N. Touzjian, C. Chutkowski, N. Kushner, M. LeFaile, L. G. Payne, and B. Roberts. 1996. Utility of SHIV for testing HIV-1 vaccine candidates in macaques. J. Acquir. Immune Defic. Syndr. Hum. Retrovirol. 12:99-106.

21. Matsuo, K., Y. Nishino, T. Kimura, R. Yamaguchi, A. Yamazaki, T. Mikami, and K. Ikuta. 1992. Highly conserved epitope domain in major core protein p24 is structurally similar among human, simian and feline immunodeficiency viruses. J. Gen. Virol. 73:2445-2450.

22. McMichael, A. J., M. Callan, V. Appay, T. Hanke, G. Ogg, and S. RowlandJones. 2000. The dynamics of the cellular immune response to HIV infection: implications for vaccination. Philos. Trans. R. Soc. Lond. B Biol. Sci. 355:1007-1011.

23. Morita, C., H. Izawa, and M. Soekawa. 1967. Isolation of a paravaccinia virus from a cow in Japan. Nippon Juigaku Zasshi 29:171-175.

24. Morita, M., Y. Aoyama, M. Arita, H. Amona, H. Yoshizawa, S. Hashizume, T. Komatsu, and I. Tagaya. 1977. Comparative studies of several vaccinia virus strains by intrathalamic inoculation into cynomolgus monkeys. Arch. Virol. 53:197-208.

25. Morita, M., M. Arita, T. Komatsu, H. Amano, and S. Hashizume. 1977. A comparison of neurovirulence of vaccinia virus by intrathalamic and/or intracisternal inoculations into cynomolgus monkeys. Microbiol. Immunol. 21:417-418.

26. Morita, M., K. Suzuki, A. Yasuda, A. Kojima, M. Sugimoto, K. Watanabe, H. Kobayashi, K. Kajima, and S. Hashizume. 1987. Recombinant vaccinia virus $\mathrm{LC} 16 \mathrm{~m} 0$ or $\mathrm{LC} 16 \mathrm{~m} 8$ that expresses hepatitis B surface antigen while preserving the attenuation of the parental virus strain. Vaccine 5:65-70.

27. Moss, B., and C. Flexner. 1987. Vaccinia virus expression vectors. Annu. Rev. Immunol. 5:305-324

28. Mothe, B. R., H. Horton, D. K. Carter, T. M. Allen, M. E. Liebl, P. Skinner, T. U. Vogel, S. Fuenger, K. Vielhuber, W. Rehrauer, N. Wilson, G. Franchini, J. D. Altman, A. Haase, L. J. Picker, D. B. Allison, and D. I. Watkins. 2002 Dominance of CD8 responses specific for epitopes bound by a single major histocompatibility complex class I molecule during the acute phase of viral infection. J. Virol. 76:875-884

29. Nabel, G. J. 2001. Challenges and opportunities for development of an AIDS vaccine. Nature 410:1002-1007.

30. Ourmanov, I., C. R. Brown, B. Moss, M. Carroll, L. Wyatt, L. Pletneva, S. Goldstein, D. Venzon, and V. M. Hirsch. 2000. Comparative efficacy of recombinant modified vaccinia virus Ankara expressing simian immunodeficiency virus (SIV) Gag-Pol and/or Env in macaques challenged with pathogenic SIV. J. Virol. 74:2740-2751.

31. Reimann, K. A., J. T. Li, G. Voss, C. Lekutis, K. Tenner-Racz, P. Racz, W. Lin, D. C. Montefiori, D. E. Lee-Parritz, Y. Lu, R. G. Collman, J. Sodroski, and N. L. Letvin. 1996. An env gene derived from a primary human immunodeficiency virus type 1 isolate confers high in vivo replicative capacity to chimeric simian/human immunodeficiency virus in rhesus monkeys. J. Virol. 70:3198-3206. 
32. Rosenberg, E. S., J. M. Billingsley, A. M. Caliendo, S. L. Boswell, P. E. Sax, S. A. Kalams, and B. D. Walker. 1997. Vigorous HIV-1-specific CD4 ${ }^{+}$T-cel responses associated with control of viremia. Science 278:1447-1450.

33. Rosenberg, E. S., L. LaRosa, T. Flynn, G. Robbins, and B. D. Walker. 1999 Characterization of HIV-1-specific T-helper cells in acute and chronic infection. Immunol. Lett. 66:89-93.

34. Sasaki, Y., Y. Ami, T. Nakasone, K. Shinohara, E. Takahashi, S. Ando, K Someya, Y. Suzaki, and M. Honda. 2000. Induction of CD95 ligand expression on $\mathrm{T}$ lymphocytes and $\mathrm{B}$ lymphocytes and its contribution to apoptosis of CD95-upregulated CD4 ${ }^{+} \mathrm{T}$ lymphocytes in macaques by infection with a pathogenic simian/human immunodeficiency virus. Clin. Exp. Immunol. 122: 381-389.

35. Seth, A., I. Ourmanov, M. J. Kuroda, J. E. Schmitz, M. W. Carroll, L. S. Wyatt, B. Moss, M. A. Forman, V. M. Hirsch, and N. L. Letvin. 1998 Recombinant modified vaccinia virus Ankara-simian immunodeficiency virus gag pol elicits cytotoxic $\mathrm{T}$ lymphocytes in rhesus monkeys detected by major histocompatibility complex class I/peptide tetramer. Proc. Natl. Acad. Sci. USA 95:10112-10116.

36. Seth, A., I. Ourmanov, J. E. Schmitz, M. J. Kuroda, M. A. Lifton, C. E Nickerson, L. Wyatt, M. Carroll, B. Moss, D. Venzon, N. L. Letvin, and V. M. Hirsch. 2000. Immunization with a modified vaccinia virus expressing simian immunodeficiency virus (SIV) Gag-Pol primes for an anamnestic Gag-specific cytotoxic T-lymphocyte response and is associated with reduction of viremia after SIV challenge. J. Virol. 74:2502-2509.

37. Shinohara, K., K. Sakai, S. Ando, Y. Ami, N. Yoshino, E. Takahashi, K. Someya, Y. Suzaki, T. Nakasone, Y. Sasaki, M. Kaizu, Y. Lu, and M. Honda. 1999. A highly pathogenic simian/human immunodeficiency virus with genetic changes in cynomolgus monkey. J. Gen. Virol. 80:1231-1240.

38. Shiver, J. W., T. M. Fu, L. Chen, D. R. Casimiro, M. E. Davies, R. K. Evans, Z. Q. Zhang, A. J. Simon, W. L. Trigona, S. A. Dubey, L. Huang, V. A. Harris, R. S. Long, X. Liang, L. Handt, W. A. Schleif, L. Zhu, D. C. Freed, N. V. Persaud, L. Guan, K. S. Punt, A. Tang, M. Chen, K. A. Wilson, K. B. Collins, G. J. Heidecker, V. R. Fernandez, H. C. Perry, J. G. Joyce, K. M. Grimm, J. C. Cook, P. M. Keller, D. S. Kresock, H. Mach, R. D. Troutman, L. A. Isopi, D. M. Williams, Z. Xu, K. E. Bohannon, D. B. Volkin, D. C. Montefiori, A. Miura, G. R. Krivulka, M. A. Lifton, M. J. Kuroda, J. E. Schmitz,
N. L. Letvin, M. J. Caulfield, A. J. Bett, R. Youil, D. C. Kaslow, and E. A. Emini. 2002. Replication-incompetent adenoviral vaccine vector elicits effective anti-immunodeficiency virus immunity. Nature 415:331-335.

39. Stahl-Hennig, C., G. Voss, U. Dittmer, C. Coulibaly, H. Petry, B. Makoschey, M. P. Cranage, A. M. Aubertin, W. Luke, and G. Hunsmann. 1993. Protection of monkeys by a split vaccine against SIVmac depends upon biological properties of the challenge virus. AIDS 7:787-795.

40. Stickl, H., V. Hochstein-Mintzel, and H. C. Huber. 1973. Primary vaccination against smallpox after preliminary vaccination with the attenuated vaccinia virus strain MVA and the use of a new "vaccination stamp." Munch. Med. Wochenschr. 115:1471-1473. (In German.)

41. Tagaya, I., T. Kitamura, and Y. Sano. 1961. A new mutant of dermovaccinia virus. Nature 192:381-382.

42. Tartaglia, J., M. E. Perkus, J. Taylor, E. K. Norton, J. C. Audonnet, W. I. Cox, S. W. Davis, J. van der Hoeven, B. Meignier, M. Riviere, et al. 1992 NYVAC: a highly attenuated strain of vaccinia virus. Virology 188:217-232.

43. Walker, B. D., and P. J. Goulder. 2000. AIDS: escape from the immune system. Nature 407:313-314.

44. Walker, B. D., and E. S. Rosenberg. 2000. Containing HIV after infection. Nat. Med. 6:1094-1095.

45. Walker, B. D., E. S. Rosenberg, C. M. Hay, N. Basgoz, and O. O. Yang. 1998 Immune control of HIV-1 replication. Adv. Exp. Med. Biol. 452:159-167.

46. Yoshino, N., Y. Ami, K. Someya, S. Ando, K. Shinohara, F. Tashiro, Y. Lu, and M. Honda. 2000. Protective immune responses induced by a non-pathogenic simian/human immunodeficiency virus (SHIV) against a challenge of a pathogenic SHIV in monkeys. Microbiol. Immunol. 44:363-372.

47. Yoshino, N., T. Ryu, M. Sugamata, T. Ihara, Y. Ami, K. Shinohara, F. Tashiro, and M. Honda. 2000. Direct detection of apoptotic cells in peripheral blood from highly pathogenic SHIV-inoculated monkey. Biochem. Biophys. Res. Commun. 268:868-874.

48. Zhou, D., Y. Shen, L. Chalifoux, D. Lee-Parritz, M. Simon, P. K. Sehgal, L. Zheng, M. Halloran, and Z. W. Chen. 1999. Mycobacterium bovis bacille Calmette-Guerin enhances pathogenicity of simian immunodeficiency virus infection and accelerates progression to AIDS in macaques: a role of persistent T-cell activation in AIDS pathogenesis. J. Immunol. 162:2204-2216. 